\title{
Late-onset programmed cell death protein-1 inhibitor-induced pneumonitis after cessation of nivolumab or pembrolizumab in patients with advanced non-small cell lung cancer: a case series
}

\author{
Hideharu Kimura ${ }^{1}$, Takashi Sone ${ }^{1}$, Tomoyuki Araya ${ }^{2}$, Akari Murata ${ }^{1}$, Kenta Yamamura ${ }^{1}$, \\ Noriyuki Ohkura ${ }^{1}$, Johsuke Hara ${ }^{1}$, Miki Abo ${ }^{1}$, Kazuo Kasahara ${ }^{1}$ \\ ${ }^{1}$ Respiratory Medicine, Kanazawa University Hospital, Ishikawa, Japan; ${ }^{2}$ Department of Respiratory Medicine, National Hospital Organization \\ Kanazawa Medical Center, Kanazawa, Japan \\ Correspondence to: Hideharu Kimura. Respiratory Medicine, Kanazawa University Hospital, Takara-machi 13-1, Kanazawa, Ishikawa 920-8641, Japan. \\ Email: hkimura3625@staff.kanazawa-u.ac.jp.
}

\begin{abstract}
Awareness of the immune-related adverse event of programmed cell death protein-1 (PD-1) inhibitor-induced pneumonitis is important. Herein, we report the clinical course of 3 patients suspected to have PD-1 inhibitor-induced pneumonitis after cessation of PD-1 inhibitor treatment. In case 1, a 62-year-old man was diagnosed with stage IVA adenocarcinoma. Nivolumab monotherapy was prescribed as secondline therapy and later discontinued due to financial reasons. Seven months after the final administration of nivolumab, the patient developed what we diagnosed as nivolumab-induced pneumonitis. The patient was immediately prescribed prednisolone $(1 \mathrm{mg} / \mathrm{kg}$ p.o. daily), and the pneumonitis resolved after 1.5 months. In case 2, a 68-year-old man was diagnosed with stage IVB squamous cell carcinoma. Nivolumab monotherapy was prescribed as fourth-line therapy. After the second administration of nivolumab, the patient developed what we diagnosed as nivolumab-induced pneumonitis; nivolumab was discontinued, and the patient was immediately prescribed prednisolone $(1 \mathrm{mg} / \mathrm{kg}$ p.o. daily). Eight months after the final administration of nivolumab, the patient again developed nivolumab-induced pneumonitis. The pneumonitis resolved without additional medication. In case 3, a 69-year-old man was diagnosed with stage IVB adenocarcinoma. Pembrolizumab monotherapy was initiated as sixth-line therapy, and it was discontinued after 4 cycles due to disease progression. Four months after the final dose of pembrolizumab, the patient developed what we diagnosed as pembrolizumab-induced pneumonitis. The patient immediately received a high intravenous dose of methylprednisolone (1,000 $\mathrm{mg}$ per day for three days). The pneumonitis and respiratory failure progressed, and he died 8 weeks after the onset of the pneumonitis. We report pneumonitis after discontinuation of ICIs in 3 patients. We confirm that, although uncommon, PD-1 inhibitor-induced irAEs can develop after treatment discontinuation. Further accumulation of cases and clarification of the clinical features of patients with irAEs, such as the time of onset, imaging findings, and treatment outcomes are needed.
\end{abstract}

Keywords: Nivolumab; pembrolizumab; drug-induced pneumonitis; immune-related adverse events (irAEs); case report

Submitted Apr 20, 2020. Accepted for publication Jan 06, 2021.

doi: $10.21037 /$ tlcr-20-582

View this article at: http://dx.doi.org/10.21037/tlcr-20-582

\section{Introduction}

This retrospective analysis of data collected from patients who received ICIs was conducted according to the protocol approved by the Institutional Review Board of Kanazawa
University Graduate School of Medicine (approved \# 2423 and \#2558) and was conducted in accordance with the Declaration of Helsinki (as revised in 2013). Written informed consent for the publication of clinical data and 
images was obtained from patient cases 1 and 2. For case 3, oral consent was obtained from his own before his death.

Recently, programmed cell death protein-1 (PD-1) and programmed death ligand-1 (PD-L1) immune checkpoint inhibitors (ICIs) have become standard treatments for a variety of cancers, including advanced non-small cell lung cancer (NSCLC). However, these agents have different toxicity profiles compared with conventional cytotoxic agents; an important issue with the ICIs is immune-related adverse events (irAEs), which are caused by activation of the immune system in multiple organs. Although ICI-induced pneumonitis (ICI-P) is not frequently observed in patients with lung cancer (1-6\%), ICI-P is the leading cause of ICIrelated death (1). The time to onset of ICI-P reported in large clinical trials of advanced NSCLC was shown to vary between 2.9 and 7.7 weeks (1). Herein, we report the clinical course of 3 patients suspected to have PD-1 inhibitorinduced pneumonitis 4,7 and 8 months after cessation of treatment, respectively. We present the following cases in accordance with the CARE reporting checklist (available at http://dx.doi.org/10.21037/tlcr-20-582).

\section{Case presentation}

\section{Case 1}

A 62-year-old Japanese man who had a 40 pack-year smoking history was diagnosed with stage IVA adenocarcinoma with multiple lung metastases in April 2015. The patient first underwent 4 cycles of combination therapy with carboplatin (AUC $=6.0$, every 3 weeks) and pemetrexed $\left(500 \mathrm{mg} / \mathrm{m}^{2}\right.$, every 3 weeks), followed by pemetrexed maintenance therapy, and a partial response was achieved. Pemetrexed maintenance therapy was discontinued due to the occurrence of pneumonitis after 5 cycles in December 2015. The pemetrexed-induced pneumonitis improved after steroid treatment. Nivolumab monotherapy (240 mg, every 2 weeks) was initiated as second-line therapy in April 2016. Although treatment resulted in stable disease, nivolumab was discontinued due to financial reasons in June 2016. After that, the patient received no treatment with antitumor agents. Seven months after the final administration of nivolumab, the patient experienced dyspnea on effort, and in January 2017, computed tomography (CT) revealed ground-glass opacities involving the upper lobe of the left lung (Figure 1). We collected bronchoalveolar lavage fluid (BALF) from the upper lobe of the left lung, and the BALF analysis revealed lymphocyte-predominant inflammatory changes. The patient's KL-6 and SP-D levels were slightly elevated compared to those before nivolumab treatment. The patient had no addition of or change in other medications after the initiation of nivolumab. We suggest that these results are consistent with a diagnosis of nivolumab-induced pneumonitis. The patient was immediately prescribed prednisolone ( $1 \mathrm{mg} / \mathrm{kg}$ p.o. daily). The pneumonitis resolved after 1.5 months (Figure 1), and the prednisolone was gradually tapered without recurrence of pneumonitis.

\section{Case 2}

A 68-year-old Japanese man who had a 90 pack-year smoking history was diagnosed with stage IVB squamous cell carcinoma with a lymph node metastasis in November 2013. The patient first underwent 5 cycles of chemotherapy with carboplatin (AUC $=6.0$, every 3 weeks) and paclitaxel $\left(210 \mathrm{mg} / \mathrm{m}^{2}\right.$, every 3 weeks). Although the tumor remained stable on this regimen, it was discontinued because the patient developed pneumonitis. After resolution of the pneumonitis, the patient underwent second-line chemotherapy with vinorelbine monotherapy $\left(25 \mathrm{mg} / \mathrm{m}^{2}\right.$, day 1,8 , every 3 weeks), followed by 2 cycles of third-line chemotherapy with carboplatin (AUC $=5.0$, every 3 weeks) and S-1 $\left(40 \mathrm{mg} / \mathrm{m}^{2}\right.$, twice daily, day 1-14, every 3 weeks), followed by S-1 alone after an allergic reaction during carboplatin administration. Nivolumab (240 mg, every 2 weeks) was initiated as fourthline therapy in May 2016. After the second administration of nivolumab, the patient developed ground-glass opacities involving a large area of the right lung on CT imaging (Figure 1). Because the findings indicated the onset of nivolumab-induced pneumonitis, nivolumab was discontinued, and the patient was immediately prescribed prednisolone ( $1 \mathrm{mg} / \mathrm{kg}$ p.o. daily). The pneumonitis resolved after 2 weeks, and the prednisolone was gradually tapered without recurrence of pneumonitis. The tumor in his right lung decreased gradually in size without any additional treatment for lung cancer. Eight months after the final administration of nivolumab, CT images revealed groundglass opacities involving the bilateral lung in February 2017 (Figure 1). Analysis of BALF collected from the upper lobe of the left lung revealed lymphocyte-predominant inflammatory changes. The patient's KL-6 and SP-D levels were elevated compared to those before nivolumab treatment. The patient had no addition of or change in other medications after the initiation of nivolumab. We suggest that these results are consistent with a diagnosis of nivolumab-induced pneumonitis. The patient had no symptoms associated with 
Case 1
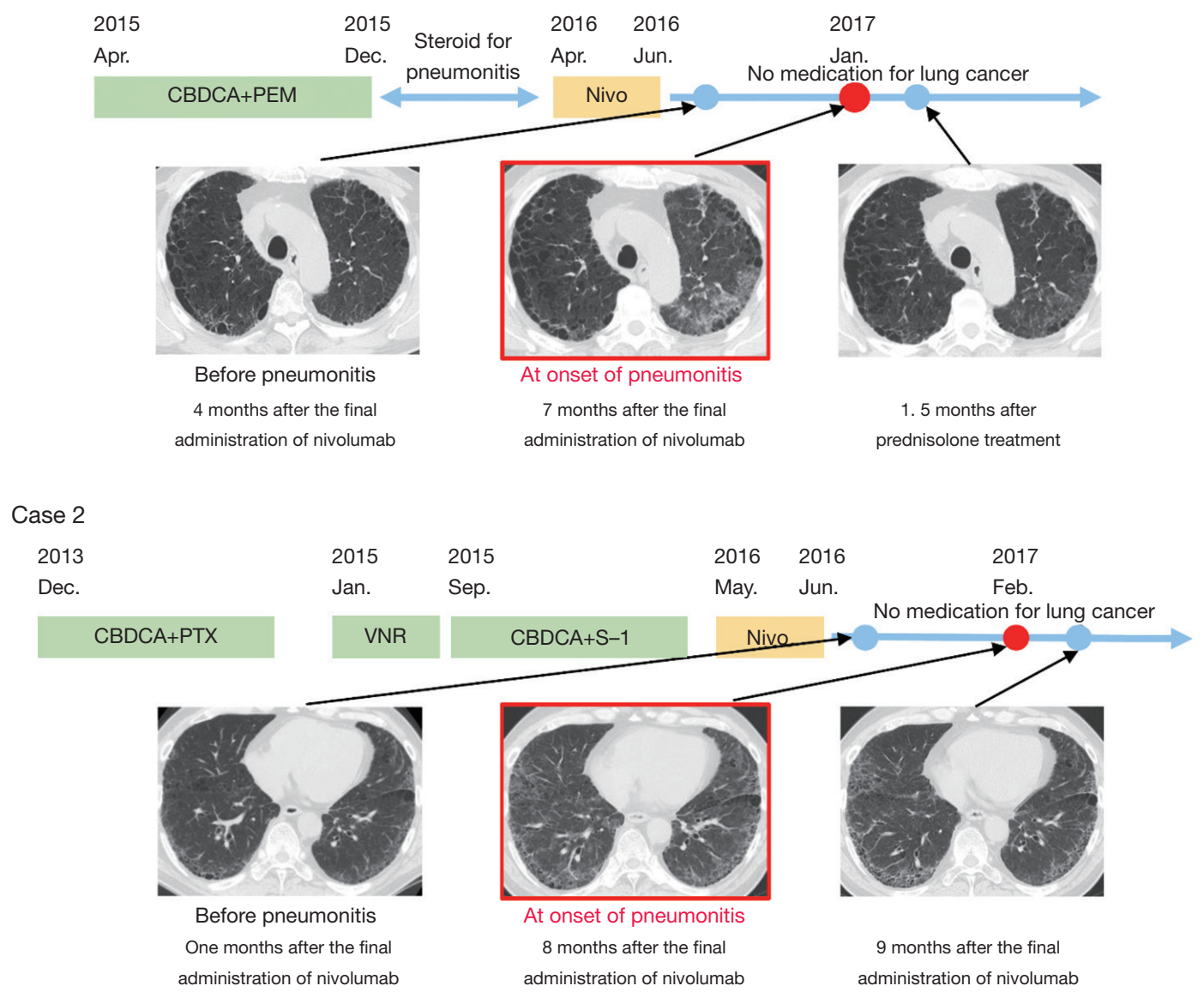

Case 3

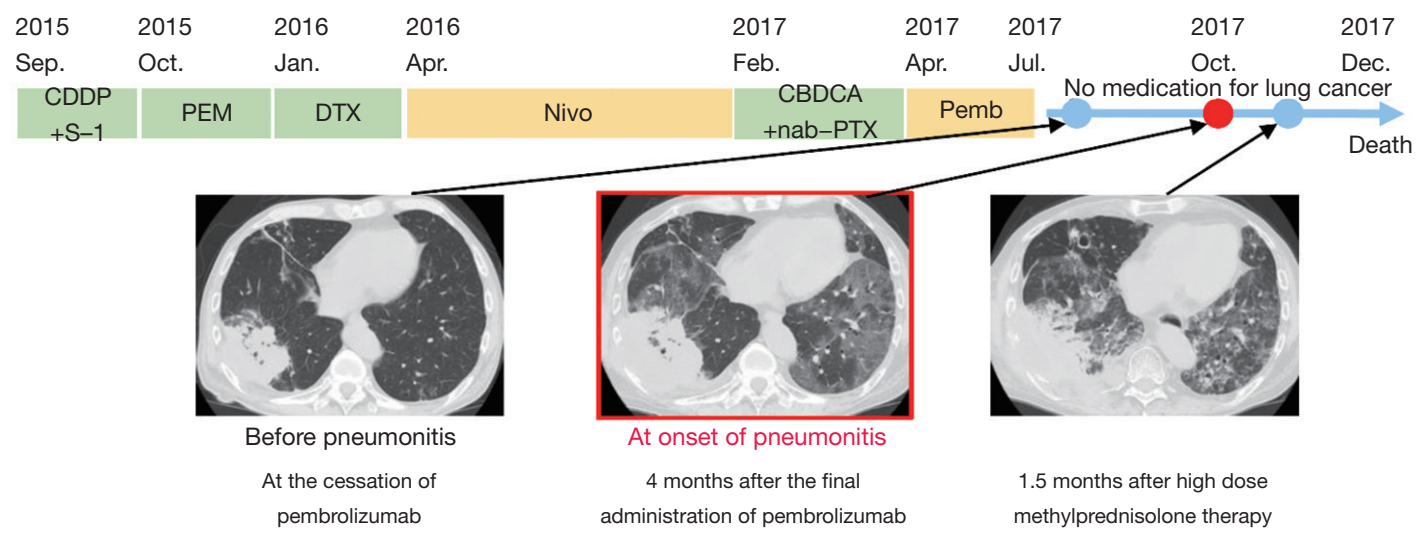

Figure 1 Clinical course of drugs administered for lung cancer and CT images. Representative images before the onset of pneumonitis, at the diagnosis of pneumonitis and after the resolution of the pneumonitis are presented. In case 1, ground-glass opacities in the left lung improved after prednisolone treatment (image on the right, as compared to that in the middle). In case 2, slight ground-glass opacities in both lung fields improved without any medication (image on the right, as compared to that in the middle). In case 3, ground-glass opacities in both lung fields failed to improve even after high-dose methylprednisolone treatment, with contraction of the lung volume (image on the right, as compared to that in the middle). 
pneumonitis, and the ground-glass opacities resolved without additional medications.

\section{Case 3}

A 69-year-old Japanese man who had a 60 pack-year smoking history was diagnosed with stage IVB adenocarcinoma with a lymph node metastasis and multiple brain metastases in September 2015. The patient first underwent a cycle of combination therapy with cisplatin $\left(60 \mathrm{mg} / \mathrm{m}^{2}\right.$, every 3 weeks) and S-1 (40 mg/m², twice daily, day 1-14, every 3 weeks), followed by pemetrexed $\left(500 \mathrm{mg} / \mathrm{m}^{2}\right.$, every 3 weeks), docetaxel (60 mg/m², every 3 weeks), nivolumab (240 mg, every 2 weeks) and a combination of carboplatin (AUC =6.0, every 4 weeks) and nanoparticle albuminbound paclitaxel (100 mg/m², day $1,8,15$, every 4 weeks). Pembrolizumab monotherapy (200 mg, every 3 weeks) was initiated as sixth-line therapy in April 2017, but it was discontinued after 4 cycles due to the progression of brain metastases. After that, the patient received supportive care alone, with no treatment with anti-tumor agents. Four months after the final administration of pembrolizumab, his respiratory failure progressed, and CT images revealed ground-glass opacities involving the bilateral lung in October 2017 (Figure 1). Due to the CT images and to the fact that the patient had no additional medications or change in other medications after the initiation of pembrolizumab, we suggest that these results are consistent with a diagnosis of pembrolizumab-induced pneumonitis. He immediately received a high intravenous dose of methylprednisolone (1000 mg per day for three days). The pneumonitis and respiratory failure progressed, and he died 8 weeks after the onset of the pneumonitis. The patient's KL-6 and SP-D levels were elevated remarkably compared to those before pembrolizumab treatment. The BALF was not analyzed.

\section{Blood concentrations of nivolumab in Case 1}

Blood samples were collected from Case 1 with written informed consent and ethical board approval from Kanazawa University (No. 344-1). Serum samples were isolated by centrifugation at $700 \times g$ for $10 \mathrm{~min}$ and were then either used immediately or stored at $-80{ }^{\circ} \mathrm{C}$ until use. Blood concentrations of nivolumab were measured using an ELISA assay, BioSim ${ }^{\mathrm{TM}}$ Nivolumab (Opdivo ${ }^{\circledR}$ ) (Human) ELISA Kit (BioVision, Milpitas, CA, USA). The blood samples were collected at the following 4 time points: before nivolumab administration (April 2016), just before the cessation of nivolumab treatment (June 2016), 2 months after the cessation of nivolumab treatment (August 2016) and at the onset of pneumonitis (January 2017). The concentrations of nivolumab at these times were under the detectable limit, 26.2, 7.7 $\mu \mathrm{g} / \mathrm{mL}$ and under the detectable limit, respectively. Although the concentration was elevated during nivolumab treatment (just before cessation) as expected, it was under the detectable limit at the onset of pneumonitis.

\section{Discussion}

Herein, we present a three-case series in which pneumonitis caused by nivolumab or pembrolizumab, both classified as PD-1 inhibitors, was strongly suspected after cessation of treatment with the drug. In the two patients in whom pneumonitis due to nivolumab therapy was suspected (cases 1 and 2), BALF analysis was performed, which revealed lymphocyte predominance, a finding consistent with drug-induced pneumonitis. As no additional therapy had been prescribed besides nivolumab or pembrolizumab in any of the three patients, the possibility of other druginduced pneumonitis was ruled out.

Reports of late-onset drug-induced pneumonitis after discontinuation of the causative drug are rare. Diamantopoulos et al. reported a patient with melanoma who developed ICI-P 6 months after discontinuation of nivolumab therapy. BALF analysis revealed lymphocyte predominance, and the ICI-P resolved with prednisolone therapy (2). The clinical course of this patient was comparable to that of case 1 of our series. As for irAEs other than pneumonitis, Takeno et al. reported a case of drug-induced adrenal dysfunction that developed 6 months after discontinuation of nivolumab therapy, and Boudjemaa et al. reported another case with the same complication developing 15 months after discontinuation of pembrolizumab therapy $(3,4)$. Teramoto et al. reported a case of type 1 diabetes mellitus that developed 6 weeks after discontinuation of nivolumab therapy (5). Amiodaroneinduced pneumonitis has been reported to develop and reexacerbate after discontinuation. There have also been reported cases of recurrent drug-induced pneumonitis 8 months after discontinuation (6), similar to our report. The long half-life of amiodarone is thought to be one of the causes of post-discontinuation onset. In addition, we previously reported a case in which the tumor continued to shrink even after discontinuation of nivolumab medication (7). These reports indicate that there are cases in which the effects of PD-1 inhibitors persist even after the drugs have been 
discontinued, as reflected by the development of irAEs and/ or tumor response persisting after treatment discontinuation. The long half-life of the PD-1 antibody could be one possible explanation for these observations. To examine the potential for this, we carried out an analysis of the serum nivolumab concentrations in case 1 . The serum samples had been collected before and at the time of discontinuation of the drug, at 2 months after discontinuation of the drug, and at the onset of ICI-P (i.e., at 7 months after discontinuation of the drug). The serum levels of nivolumab were under the detection limit, 26.2, $7.7 \mu \mathrm{g} / \mathrm{mL}$, and under the detection limit, respectively, at these time points. Hence, the drug was undetectable in the serum at the onset of ICI-P, whereas it was demonstrable in the serum during nivolumab therapy. The time course of the serum levels was within the expected range; however, it did not support the hypothesis that the long half-life of nivolumab and, therefore, its persistence in the circulation even after cessation of therapy may have had a bearing on the development of irAEs after discontinuation of the drug.

To our knowledge, this is the first report to show in actual clinical cases that PD-1 inhibitors can induce pneumonitis after discontinuation, especially months after discontinuation, although it has some limitations. First, the definitive diagnosis of drug-induced pneumonia is often difficult in actual clinical situations. In case 1 and case 2, BALF findings were helpful for the diagnosis of the nivolumab-induced pneumonitis. It is well known that BALF findings in drug-induced pneumonitis are predominant in lymphocytes. In a study of patients with immune-checkpoint inhibitor-induced pneumonitis, the results of BALF analysis showed lymphocyte alveolitis. Delaunay et al. showed that BALF indicated T-lymphocytic alveolitis in 24 of 35 cases (8). Additionally, Cho et al. performed BALF analysis in five patients and found a median lymphocyte proportion of $14.0 \%$ (4.0-90.0\%) in the lavage fluid (9). A subset of idiopathic interstitial pneumonia, including idiopathic non-specific interstitial pneumonia and cryptogenic organizing pneumonia and connective tissue disorder-related lung disease, is also predominant in lymphocytes and cannot be distinguished by only BALF findings. However, since infectious disease, malignant disease, heart failure and alveolar hemorrhage can be excluded, BALF analysis plays an important role in supporting the diagnosis of drug-induced pneumonitis. In case 3, BALF analysis could not be performed due to respiratory failure. Because BALF collection has the risk of exacerbating respiratory failure, we often decide not to perform it in patients who are in poor clinical condition.
Since BALF analysis findings were lacking, the diagnostic support was weaker than in the other 2 cases, but the condition was diagnosed clinically to be consistent with pembrolizumab-induced pneumonitis. It is even more difficult to identify the causative drug. In our cases as well, drug-induced pneumonitis was suspected by excluding other lung disorders, such as infections, and in cases 1 and 2, there was no other suspicious drug, so they were diagnosed as nivolumab-induced pneumonitis. In case 3 , other drugs were administered, but pembrolizumab-induced pneumonitis was most suspected based on the experience of using the drug in this case and the likelihood of druginduced pneumonitis. Moreover, the reason it develops after treatment discontinuation remains unclear. Persistence of high blood levels, such as with amiodarone, was considered as one possibility, but the ELISA analysis of blood levels of nivolumab that we performed did not support this explanation, as described above.

\section{Conclusions}

The present report describes the development of ICI-P after treatment discontinuation in 3 patients. Considering that other such cases have also been reported previously, we confirm that PD-1 inhibitor-related irAEs can develop, although they are rather uncommon, after discontinuation of the drugs. Further accumulation of cases and clarification of the clinical features of patients with irAEs, such as the time of onset, imaging findings, and treatment outcomes are needed.

\section{Acknowledgments}

Funding: None.

\section{Footnote}

Reporting Checklist: The authors have completed the CARE reporting checklist. Available at http://dx.doi.org/10.21037/ tlcr-20-582

Conflicts of Interest: All authors have completed the ICMJE uniform disclosure form (available at http://dx.doi. org/10.21037/tlcr-20-582). Dr. Kimura reports personal fees from Behringer Ingelheim, grants and personal fees from Pfizer, personal fees from MSD Japan, outside the submitted work; Dr. Hara reports personal fees from AstraZeneca, personal fees from Novartis, personal fees 
from GlaxoSmithKline, personal fees from TAIHO PHARMACEUTICAL, outside the submitted work; Dr. Kasahara reports grants, personal fees and other from AstraZeneca, grants, personal fees and other from Chugai Pharmaceuticals Co,. LTD., grants, personal fees and other from Bristol-Myers Squibb, grants, personal fees and other from MSD, grants, personal fees and other from Eli Lilly and Company, grants, personal fees and other from Boehringer Ingelheim, grants and other from Ono Pharmaceutical Co., grants, personal fees and other from Taiho Pharma, outside the submitted work. The other authors have no conflicts of interest to declare.

Ethical Statement: The authors are accountable for all aspects of the work in ensuring that questions related to the accuracy or integrity of any part of the work are appropriately investigated and resolved. This retrospective analysis of data collected from patients who received ICIs was conducted according to the protocol approved by the Institutional Review Board of Kanazawa University Graduate School of Medicine (approved \# 2423 and \#2558) and was conducted in accordance with the Declaration of Helsinki (as revised in 2013). Written informed consent for the publication of clinical data and images was obtained from patient cases 1 and 2. For case 3, oral consent was obtained from his own before his death.

Open Access Statement: This is an Open Access article distributed in accordance with the Creative Commons Attribution-NonCommercial-NoDerivs 4.0 International License (CC BY-NC-ND 4.0), which permits the noncommercial replication and distribution of the article with the strict proviso that no changes or edits are made and the original work is properly cited (including links to both the formal publication through the relevant DOI and the license). See: https://creativecommons.org/licenses/by-nc-nd/4.0/.

\section{References}

1. Cadranel J, Canellas A, Matton L, et al. Pulmonary complications of immune checkpoint inhibitors in patients with nonsmall cell lung cancer. Eur Respir Rev 2019;28:190058.

2. Diamantopoulos PT, Gaggadi M, Kassi E, et al. Lateonset nivolumab-mediated pneumonitis in a patient with melanoma and multiple immune-related adverse events. Melanoma Res 2017;27:391-5.

3. Takeno A, Yamamoto M, Morita M, et al. Late-onset isolated adrenocorticotropic hormone deficiency caused by nivolumab: A case report. BMC Endocr Disord 2019;19:25.

4. Boudjemaa A, Rousseau-Bussac G, Monnet I. Late-Onset Adrenal Insufficiency More Than 1 Year after Stopping Pembrolizumab. J Thorac Oncol 2018;13:e39-40.

5. Teramoto $Y$, Nakamura $Y$, Asami $Y$, et al. Case of type 1 diabetes associated with less-dose nivolumab therapy in a melanoma patient. J Dermatol 2017;44:605-6.

6. Chendrasekhar A, Barke RA, Druck P. Recurrent amiodarone pulmonary toxicity. South Med J 1996;89:85-6.

7. Kimura H, Sone T, Murata A, et al. Long-lasting shrinkage in tumor mass after discontinuation of nivolumab treatment. Lung Cancer 2017;108:7-8.

8. Delaunay M, Cadranel J, Lusque A, et al. Immunecheckpoint inhibitors associated with interstitial lung disease in cancer patients. Eur Respir J 2017;50:1700050.

9. Cho JY, Kim J, Lee JS, et al. Characteristics, incidence, and risk factors of immune checkpoint inhibitor-related pneumonitis in patients with non-small cell lung cancer. Lung Cancer 2018;125:150-6.
Cite this article as: Kimura H, Sone T, Araya T, Murata A, Yamamura K, Ohkura N, Hara J, Abo M, Kasahara K. Lateonset programmed cell death protein-1 inhibitor-induced pneumonitis after cessation of nivolumab or pembrolizumab in patients with advanced non-small cell lung cancer: a case series. Transl Lung Cancer Res 2021;10(3):1576-1581. doi: 10.21037/ tlcr-20-582 